\title{
Laboratory technologies in independent medical laboratories and analysis of its resource cost
}

\author{
Zhen Tian \\ Zhengzhou University of Light Industry, \\ Zhengzhou, Henan, 450002, \\ China
}

Received: September 8, 2020. Revised: November 16, 2020. Accepted: November 18, 2020. Published: November 19, 2020.

\begin{abstract}
Medical laboratory technology is an important basis for clinical diagnosis and treatment. However, due to problems such as idle resources and high testing costs in laboratories of large hospitals, independent medical laboratory emerged. The independent medical laboratory can independently perform medical tests outside the hospital and can effectively share resources, with low cost and strong specificity. In this study, the advantages of an independent medical laboratory were analyzed to suggest its significance, then the laboratory technologies and resource costs in laboratories were analyzed, some suggestions were put forward for improving technologies and reducing costs, and finally the prospects for the development of the independent medical laboratory was discussed briefly. This work has values for promoting the good development of independent laboratory and the further development of the medical market.
\end{abstract}

Keywords-Cost analysis, independent medical laboratory, laboratory technology.

\section{INTRODUCTION}

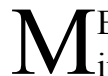
EDICAL laboratory technology plays an important role in clinical diagnosis and treatment. Currently the laboratory testing in China has problems such as repeated purchasing, repeated testing, high cost and low level. But cost saving and high technical level are the advantages of independent medical laboratory [1]. The independent medical laboratory is an institution which has independent legal personality approved by the administrative department of health and is responsible for testing human body specimens [2]. With the deepening of the reform of the medical system, the independent medical laboratory has been getting better and better. The hierarchical diagnosis and treatment also provides a good condition for the development of independent medical laboratories [3].

Through surveying the basic medical service institutions in Chongqing, China, Weng et al. [4] came up with the idea of establishing independent medical laboratories and considered that independent medical laboratory could also relieve manpower and financial pressure besides improving the service level. Song [5] suggested monitoring independent medical laboratory in aspects of management, quality, benefit and safety for the current defects and bugs of independent medical laboratories. Zhao et al. [6] established a third party medical laboratory informatization platform which included 19 hospitals and a independent medical laboratory to share the test results of different medical institutions and promote the improvement of skills and services. Luo [7] chose an independent medical laboratory for cooperation by means of invitation for bid and found that independent medical laboratories could help improve the diagnosis and treatment levels of clinical departments and reduce cost. Lin et al. [8] advocated establishing the third-party medical laboratory institution with international test skills by innovating the business mode and logistic transportation. Natalia et al. [9] analyzed the diagnosis cost and research cost in the medical laboratory in Russian and searched the method of improving medical healthcare from the perspective of cost implementation. Hueth et al. [10] pointed out that unnecessary repeated testing in medical institutions led to many unnecessary health care expenses. In the analysis of all inpatient orders, $44 \%$ of repeated tests were found, and the cost was about 37,376 US dollars. The independent medical laboratory's development in China started with the establishment of Guangzhou Jinyu Medical Testing Center in 
1994 and is now in the stage of rapid development. However, compared with developed countries such as Europe and America, the development level of the independent medical laboratory in China is still low. The inspection income of the independent medical laboratory accounts for only $1.5 \%$ of medical examination, and the test items are few. However, in terms of the current development situation, China's independent medical laboratory has great development potential. Therefore, China should learn from the successful experience of foreign countries and vigorously develop the independent medical laboratory on the premise of considering China's actual situation. At present, there is little research on the independent medical laboratory, and there is also a lack of targeted analysis on its technology and cost. Therefore, this study analyzed the advantages and values of the independent medical laboratory from aspects of detection technology and cost analysis through material collection and data analysis and put forward some suggestions for its future development, so as to promote the better and faster development of the independent medical laboratory.

\section{SignifiCANCE OF ESTABLISHING INDEPENDENT MEDICAL LABORATORIES}

Laboratory testing in China is mainly carried out in laboratory department of hospitals, but there are some problems in its development.

The first problem is idleness of equipment. Most of the laboratory departments in large hospitals are fully equipped, but the utilization frequency of the equipment is low, resulting in waste of resources. Taking automatic biochemical tester as an example, it can complete 1000 tests per hour and fulfill the daily workload in several hours; it is idled in the rest of the time.

The second problem is the high cost. Because of the high cost of laboratory equipment, the testing charges are usually high. If there is a shift from one hospital to another, the new hospital will often require a new test, which increases testing cost and aggravates the financial burden of patients.

The last problem is the low basic testing level. Patients usually cannot be timely and accurately diagnosed in basic medical institutions because of the weak capital strength, incomplete equipment and low testing level, which can cause the loss of patients and profit reduction. As a result, the hospitals cannot afford advanced equipment.

The biggest advantage of the independent medical laboratory is that it can share resources and save the cost, suggesting a broad development prospect. Establishing independent medical laboratories has multiple disadvantages.

Firstly, it can improve the competitiveness of small and medium-sized medical institutions. Small and medium-sized medical institutions usually have low testing level and incomplete equipment; hence they are difficult to gain profits. Independent medical laboratories can provide small and medium-sized medical institutions with high-quality testing service and help them save cost.
Secondly, it can promote reasonable distribution of resources. Many large hospitals have a variety of facilities and abundant financial resources, but many resources are wasted because of the limited workload. Independent medical laboratory institutions which are equipped with high-quality equipment and talents can provide high-quality services for medical institutions in the same region, avoid the repeated purchase and idleness of equipment, and improve the utilization rate of equipment.

Thirdly, it can enhance test level. The concentration of the high-level equipment and talents can provide laboratories with better condition for studying testing technologies and developing cutting-edge technology. The large-scale test samples from various medical institutions can help laboratories further enhance test level.

Fourthly, it can reduce medical cost [11]. The demand of independent medical laboratories for equipment and reagents are significantly larger than that of single hospital. Therefore they can purchase equipment and reagents in bulk in a preferential price. The cost reduction results in lower testing charges, which can relieve the financial burden of patients. Moreover independent medical institutions can avoid repeated testing.

\section{ANALYSIS OF TESTING TECHNOLOGIES IN INDEPENDENT MEDICAL LABORATORIES}

\section{A. Laboratory Technologies in Independent Medical Laboratories}

Independent medical laboratories are featured by highquality and high-efficient testing. There are many high-level testing instruments and high-quality technical talents in independent medical laboratories. The mass concentration of samples is good for experiments. Resource sharing can avoid repeated purchase of instruments and realize some uncommon test items, for example, monitoring of plasma drug concentration. The independent medical laboratory which takes testing as the only target can only take efforts to develop scientific research ability and enhance laboratory technologies to gain larger profits.

The independent medical laboratory has the ability to carry out some special projects with higher cost and fewer specimens. On the one hand, it can improve the level of testing; on the one hand, it is also beneficial to the progress of medical technology. The laboratories can also carry out some items with social values, such as prenatal diagnosis of congenital diseases and examination of neonatal genetic diseases.

Independent medical laboratories usually centers on laboratory diagnostic departments including clinical biochemical laboratory, gene diagnosis room and cytopathological room. They are mature in medical lab test. Taking HIV antibody screening as an example, the testing flow is shown in Fig. 1. 


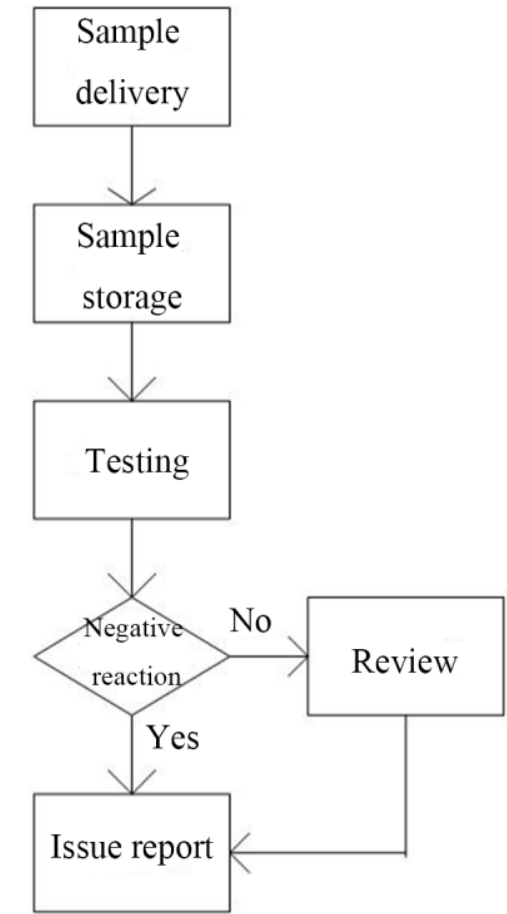

Fig. 1 The flow of screening HIV antibody

After sample collection, the sample is sent to the laboratory in the prescribed time. The barcode containing the patient's information is attached to the container of the sample. After the sample is received, the sample barcode and the sample quality are checked, and the unqualified samples are sent back. Before the test starts, we should firstly prepare the ELISA kit for detection of HIV antibody SOP and then detected according to the requirements of the instructions. After detection, a report of HIV antibody negative is issued to the sample with negative reaction, and the sample which had positive reaction are reviewed using the original reagent and the other reagent. If the results of both tests were positive, then a report of HIV antibody positive is issued.

Independent medical laboratories has a series of standard operating documents involving sample processing, instrument use and maintenance and reagent preparation, which can ensure the reliability of testing results.

Independent medical laboratories in China has some problems currently, which is not beneficial to the development of laboratories. For example, the management consciousness for laboratory equipment is weak; equipment is not repaired and maintained regularly, leading to shorter service life and low testing accuracy. Laboratory technicians are not qualified to operate laboratory equipment normatively, and the substandard technology cannot ensure testing quality. Moreover testing quality management is not rigorous. There are no specialized management and supervision institutions and relevant systems to supervise and manage laboratory technicians. To further improve the testing level of laboratories, multiple efforts are needed. Relevant government departments should provide corresponding guidance and management for the development of laboratories and make reasonable planning for the establishment of independent medical laboratories according to the actual situations of economical development and medical resources. The laboratories need to improve the technical level and management consciousness and strengthen the training of inspection personnel to realize the significant development of independent medical laboratories.

\section{B. Methods to Improve Laboratory Technologies}

Talent cultivation should be strengthened to improve their comprehensive quality. Independent medical laboratories should cooperate with medical colleges and universities [12]. For example, Zhejiang Dian Diagnostics Co., Ltd. established strategic cooperation with Hangzhou Medical College in 2016 and established Dian Inspection Institute as a talent cultivation base. Moreover, the laboratories should recruit postgraduate students, cultivate advanced laboratory technicians, encourage laboratory backbone to receive further education, and improve the quantity and quality of technicians. Various inspection training and academic activities should be carried out in forms of lecture and training to realize the comprehensive cultivation of scientific manpower. For example, laboratory technicians are informed with the use, operation method and matters needing attentions of new equipment by means of theme lecture. Reasonable allocation of laboratory staffs is also a good way. Skilled laboratory staffs can be assigned to different departments for assistance to promote the overall technical level.

Academic exchange and reward for scientific research should be paid attention to. Laboratory staffs should positively attend domestic and foreign academic conferences to learn the advanced management concepts and laboratory technologies in other independent medical laboratories. Researchers who published scientific research papers on authoritative journal should be rewarded to encourage laboratory staffs to actively attend scientific research works and improve technical level.

Operation procedures should be standardized. The sources of reagents should be unified to reduce the difference between different batches. Instrument model should also be unified. Instruments should be sent for regular quality tests which are organized by district government. Every procedure should be strictly checked. Correct operation of equipment and repeated testing are needed to improve the accuracy of results. To ensure the consistency of the inspection results, industry norms should be established, and regular assessment is needed.

It is also necessary to supervise quality and perfect management system. Supervision groups should be set up to supervise the testing process and quality. Moreover testing results were randomly checked. The substandard operations should be corrected. The internal management mechanism should be perfected. Relevant rules and regulations should be clarified. Laboratory staffs should be supervised to learn advanced technologies and improve professional level. The monitoring of the daily inspection process in laboratories should be strengthened through informatization method. The inspection quality should be taken as the main theme of the 
development.

\section{ANALYSIS ON COST OF LABORATORY RESOURCES}

\section{A. Analysis on Laboratory Cost}

Compared to hospitals, independent medical laboratories cost more. Though the investment cost and reagent cost of independent medical laboratories have price advantage because of bulk buying, the cost of such a large amount of equipment and reagents is still an extremely large cost. Moreover laboratories need to preserve and transport samples, which will also cost a lot. Taking an independent medical laboratory in Beijing as an example, there are large advanced laboratory instruments such as AU2700 fully automatic biochemical analyzer and fully automatic blood analyzer; about 50 equipment in the laboratory are worth more than 100 thousand yuan; the fixed asset value is about 50 million yuan and increasing year by year. There are more than 100 professional laboratory staffs, and the staff composition is shown in Fig. 2.

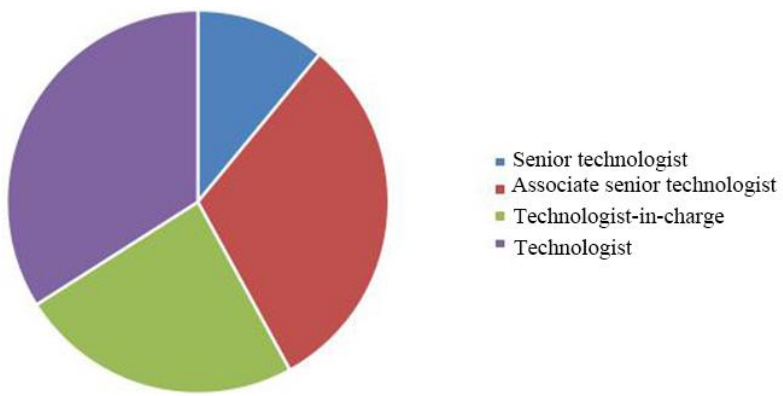

Fig. 2 The composition of laboratory staffs

The annual income and expense of the laboratory are shown in Table 1.

Table 1 The income and expense of the laboratory

\begin{tabular}{|l|l|}
\hline Item & $\begin{array}{l}\text { Amount (ten thousand } \\
\text { yuan) }\end{array}$ \\
\hline Business income & 3500 \\
\hline business expense & 1200 \\
\hline Depreciation of equipment & 250 \\
\hline Staff cost & 160 \\
\hline Total cost & 1610 \\
\hline Net income & 1890 \\
\hline
\end{tabular}

Table 2 Business income and workload

\begin{tabular}{|l|l|}
\hline Item & Amount \\
\hline Business income (10 thousand yuan) & 3500 \\
\hline Workload (10 thousand times) & 70 \\
\hline Business income per time (yuan) & 50 \\
\hline
\end{tabular}

Table 1 and 2 demonstrated that the business expense of the laboratory reached 12 million yuan, and the total expense is 16.1 million yuan after adding depreciation allowances of equipment, staff salary and welfare, which is $46 \%$ that of the annual income. The annual workload of the laboratory reached
700 thousand times, bringing an income of 35 million yuan. After the cost is reduced, the annual net income of the laboratory was 18 million 900 thousand yuan, suggesting a favourable earning performance.

The automatic blood analyzer, automatic biochemical analyzer, and automatic drug sensitivity analysis system were taken as examples. The resource costs of the three equipment were analyzed, and the results are shown in Table 3.

Table 3 The resource cost analysis of different equipment

\begin{tabular}{|l|l|l|l|}
\hline & $\begin{array}{l}\text { Automatic } \\
\text { blood } \\
\text { analyzer }\end{array}$ & $\begin{array}{l}\text { Automatic } \\
\text { biochemical } \\
\text { analyzer }\end{array}$ & $\begin{array}{l}\text { Automatic } \\
\text { drug } \\
\text { sensitivity } \\
\text { analysis } \\
\text { system }\end{array}$ \\
\hline $\begin{array}{l}\text { Operation } \\
\text { time/h }\end{array}$ & 2080 & 2426 & 4068 \\
\hline $\begin{array}{l}\text { Number of } \\
\text { detection/n }\end{array}$ & 72105 & 1512584 & 1326 \\
\hline $\begin{array}{l}\text { Business } \\
\text { income/yuan }\end{array}$ & 1325468 & 12789542 & 215485 \\
\hline $\begin{array}{l}\text { Material } \\
\text { cost/yuan }\end{array}$ & 320146 & 3254685 & 125841 \\
\hline $\begin{array}{l}\text { Maintenance } \\
\text { costs/yuan }\end{array}$ & 12520 & 32568 & 10624 \\
\hline $\begin{array}{l}\text { Equipment } \\
\text { depreciation } \\
\text { expense/yuan }\end{array}$ & 9145 & 401254 & 15000 \\
\hline $\begin{array}{l}\text { Staff } \\
\text { salary/yuan }\end{array}$ & 125241 & 456214 & 468521 \\
\hline $\begin{array}{l}\text { Net } \\
\text { income/yuan }\end{array}$ & 858416 & 8644821 & -278660 \\
\hline
\end{tabular}

The business income and net income of the three equipment were compared, shown in Fig. 3.

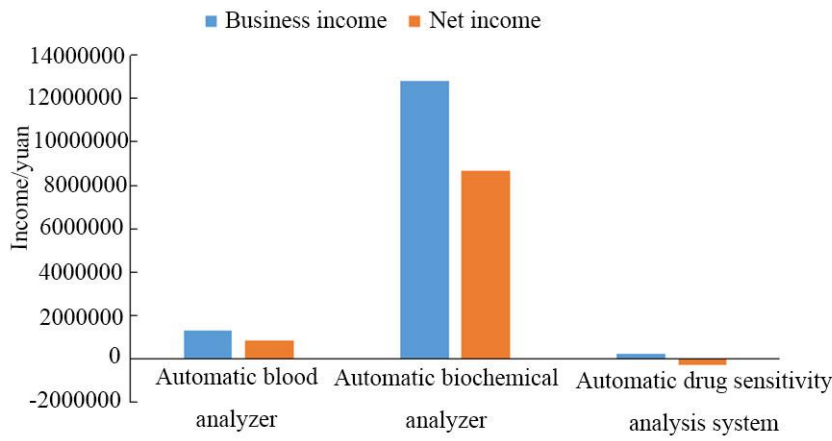

Fig. 3 The business income and net income of equipment

Table 3 and Fig. 3 show that the automatic biochemical analyzer had the largest number of detection and the largest business income, the costs of the automatic biochemical analyzer, such as material costs, maintenance costs, etc., were significantly higher than those of the other two devices, but its net income was still the highest. The operation time of the automatic blood analyzer and automatic biochemical analyzer was close, but the number of detection was less; thus, the net income was small. The operation time of the automatic drug sensitivity analysis system was the longest, but the number of 
defection was less, the income and cost were slightly small, but the salary was relatively high; thus, its net income was the lowest, which was negative. Although the economic benefit of the automatic drug sensitivity analysis system was low, it was indispensable for the laboratory in the aspect of the operation time. Generally speaking, the resource cost of the independent medical laboratory was reasonable.

\section{DISCUSSION}

Medical laboratory technology is an important step in clinical diagnosis and treatment. As a supplement to the hospital laboratory, the independent medical laboratory has unique advantages in reducing costs and improving efficiency. With the deepening of medical reform, the independent medical laboratory has been developed rapidly, but there are still some deficiencies in the detection level, cost management, etc. How to realize the optimal development of the independent medical laboratory needs further research.

In the study of the independent medical laboratory, Sun et al. [13] compared the laboratory of health care center with that of the third-party medical laboratory. It was found that although the laboratory of health care center had equipment with higher values and larger total area, its detection ability was inferior to that of the third-party medical laboratory, and there are such phenomena as repeated construction, repeated investment, and unreasonable resource allocation. Therefore, more resources should focus on the establishment of thirdparty medical laboratories to facilitate the testing work. The study of Sun et al. [13] emphasized the analysis of the laboratory of health care center and concerned little about the analysis of third-party medical laboratories. This paper focused on the research of the independent medical laboratory and put forward more suggestions for its development through the analysis of detection technology and resource cost. In order to reduce the inspection cost, the independent medical laboratory should:

(1) perfect the internal accounting system. To reduce cost, it is essential to perfect the current accounting system, establish reagent management department to purchase, check, accept, reserve and regulate reagents, extend inbound channel and formulate internal control system [14]. The service condition of reagents in laboratories should be concerned to control purchasing and storage costs. Besides, purchasing by invitation to bid can be used. The bidding procedure is strictly executed. Reagents which are inexpensive and have good quality can be purchased. Centralized bidding can avoid the problem of repeated purchasing of decentralized bidding.

(2) strengthen the maintenance of equipment. Cost of equipment maintenance takes up a large part of business expense. Paying attention to daily maintenance of equipment and protecting equipment during use can reduce the cost of maintenance. Specialized equipment maintainers can be assigned to ensure the normal operation of equipment.

(3) strengthen staff training. Staffs in independent medical laboratories should be medical practitioners who have a full understanding of reagent storage and purchasing. Currently, the reagent purchasing and testing are carried out by many non-professional staff, which is not beneficial to the test. Moreover, the staffs should be given intensive training about the dosage of reagents, operation method, and operation procedure to reduce waste of reagents. System of rewards and punishments should be implemented to control dosage of reagents and reduce cost of reagents.

(4) establish the informatization system. With the advancement of technologies, informatization approaches have gradually entered the medical system. The staff can understand the profit and loss through analyzing the revenue and expenditure of testing items with the informatization system, which has a smaller workload and a lower error rate compared to the traditional mode $[15,16]$. For example, regularly manage the equipment in laboratories through equipment file management is beneficial to prolong the service life of the equipment.

\section{CONCLUSION}

Independent medical laboratories in China have a broad development prospect though starting late. The establishment of independent medical laboratories can optimize resource allocation, enhance testing level, and reduce testing cost, which is of great significance to the development of medical market. In this study, the laboratory technologies and resource cost of independent medical laboratories were analyzed. Compared with the study of Sun et al. [13], this study focused more on the research of the independent medical laboratory, analyzed the necessity of establishing the independent medical laboratory, and made specific analyses on several equipment in the laboratory. Moreover, this study put forward some suggestions for improving laboratory technologies and reducing cost of equipment, which is beneficial to improve the technical level and reasonable cost planning in the independent medical laboratory and promote the further balanced development of the medical market.

\section{References}

[1] A. M. G. Kandilov, G. C. Pope, J. Kautter, et al., "The National Market for Medicare Clinical Laboratory Testing: Implications for Payment Reform," Medicare Medicaid Res. Rev., vol. 2, pp. 1-21, June 2012.

[2] E. Lin, Y. Liang, Z. Zhou, et al., "Discussion on the innovation practice of the independent clinical laboratories under the "internet + " age," Chin. J. Clin. Lab. Manag., no. 2, pp. 78-81, April 2016.

[3] Qi X, Shen Z, Lian Y, et al. "Analysis of the development of independent clinical laboratories under the policy of hierarchical medical system," Chin. J. Clin. Lab. Manag., no. 4, pp. 197-200, October 2016.

[4] C. G. Weng, X. Chen, and Y. G. Weng, "Necessity for the Construction of Independent Medical Laboratories," Res. Explorat. Lab., no. 3, pp. 182-184, March 2012.

[5] C. Song, "Investigation of Problems and Discussion on Supervision and Management Mode for Independent 
Clinical Laboratories in China," Chin. Hosp. Manag., vol. 18, pp. 119-128, January 2011.

[6] W. Zhao, Y. T. Cao, Y. L. Zhan, et al., "Establishment and exploration of the independent clinical laboratory service platform," Lab. Med. Clin., vol. 12, no. 20, pp. 2979-2980, October 2015.

[7] J. Luo, "The practice and experience of selection about independent medical laboratory cooperation in our hospital," Chin. J. Health Lab. Tech., no. 12, pp. 20282089, June 2015.

[8] Y. Liu, "Function of the Independent Clinical Laboratory to Improve the Current Clinical Laboratory Situation of Medical Institutions Funded by Social Capital in Pudong New Area," Med. Soc., no. 11, pp. 55-57, November 2013.

[9] N. Bryksina, A. Golovina, and F. Legotin, "Implementation of cost leadership strategy by russian medical companies of laboratory diagnostics," pp. 189198, March 2018.

[10] K. D. Hueth, B. R. Jackson, and R. L. Schmidt, "An audit of repeat testing at an academic medical centerconsistency of order patterns with recommendations and potential cost savings," Am. J. Clin. Pathol., vol. 150, no. 1, pp. 27-33, April 2018.

[11]W. B. Wang, and D. Q. Wang, "Development of Independent Medical Laboratories in the Present and Future," Labeled Immunoassays Clin. Med., vol. 23, no. 8 pp. 954-957, August 2016.

[12] Y. Mao, P. Cao, F. Pan, et al., "Thinking of current situation and problems in independent clinical laboratory technical talents training," Chin. J. Clin. Lab. Manag., vol. 5, no. 3, pp. 152-155, August 2017.

[13]X. F. Sun, L. Zhu, S. Q. Wang, et al., "Discussion on construction of third-party medical laboratory at international travel healthcare centers," Chin. J. Front. Health Quarant., vol. 39, no. 4, pp. 302-304, August 2016.

[14] C. Garcia-Hejl, D. Chianéa, E. Dedome, et al., "[Internal audit in medical laboratory: what means of control for an effective audit process?]," Ann. Biol. Clin., vol. 71, pp. 615-624, October 2013.

[15] J. Ying, Y. Z. Zhao, and S. L. He, "Development of Digital Platform for Quality Inspection of Medical Equipment," Chin. Med. Equipment J., no. 3, pp. 25-26, March 2011.

[16]H. Xu, and F. Xu, "Research on informatization construction of inspection and quality control for medical devices in hospital critical platform," China Med. Equipment, no. 4, pp. 13-16, April 2011.

[17] J. Wang, X. P. Xing, and L. J. Wang, "Equipment File Management of Independent Medical Laboratory," Chin. Med. Equipment J., no. 1, pp. 94-95, January 2010.

Zhen Tian, born in 1984, has received the doctor's degree. She is a lecturer in Zhengzhou University of Light Industry, China. She is interested in economic management.

\section{Creative Commons Attribution License 4.0 (Attribution 4.0 International, CC BY 4.0)}

This article is published under the terms of the Creative Commons Attribution License 4.0 https://creativecommons.org/licenses/by/4.0/deed.en_US 